\title{
Parent training interventions for attention deficit hyperactivity disorder
}

\author{
Morris Zwi ${ }^{1}$, Hannah Jones ${ }^{2}$, Camilla Thorgaard ${ }^{3}$, Ann York ${ }^{1}$, and Jane A Dennis ${ }^{4}$ \\ ${ }^{1}$ Richmond Royal Hospital, South West London \& St George's NHS Mental Health Trust, \\ Richmond, UK \\ 2Literature and Evidence Research Unit (LERU), Institute of Mental Health, Nottinghamshire \\ Healthcare NHS Trust, Woodbeck, UK \\ ${ }^{3}$ Kraftens Bekæmpelse, Strandboulevarden 49, København Ø, Denmark \\ ${ }^{4}$ School for Policy Studies, University of Bristol, Bristol, UK
}

\begin{abstract}
This is the protocol for a review and there is no abstract. The objectives are as follows:

To determine whether parent-training interventions are effective in reducing ADHD symptoms and associated problems (e.g. disruptive behaviour disorders or child-specific impairments such as learning difficulties) in children and young people aged 5-18 with ADHD, compared to controls with no parent-training interventions.
\end{abstract}

\section{BACKGROUND}

\section{Description of the condition}

Definition and prevalence of Attention Deficit Hyperactivity Disorder-Attention

Deficit Hyperactivity Disorder (ADHD) is a neurodevelopmental disorder characterised by high levels of inattention, hyperactivity and impulsivity present before seven years of age, seen in a range of situations, inconsistent with the developmental level of the child and associated with impairment in social or academic development (APA 1994). In the International Classification of Diseases (ICD-10) (WHO 1992) Hyperkinetic Disorder

Copyright () 2009 The Cochrane Collaboration. Published by John Wiley \& Sons, Ltd.

Contact address: Morris Zwi, Richmond Royal Hospital, South West London \& St George's NHS Mental Health Trust, Kew Foot Road, Richmond, Surrey, TW92TE, UK. mzwi@btinternet.com. morris.zwi@swlstg-tr.nhs.uk.

CONTRIBUTIONS OF AUTHORS

Morris Zwi (MZ) conceived and designed original review question and wrote the background of the protocol with assistance from Ann York (AY). Jane Dennis (JD), Hannah Jones (HJ) and Camilla Thorgaard (CT) contributed to the refining of inclusion criteria. $\mathrm{MZ}, \mathrm{JD}$ and $\mathrm{HJ}$ wrote the Methods sections.

Searches will be run by Jo Abbott (TSC, CDPLPG) and results will be vetted in pairs by MZ, JD and HJ. Studies will be assessed for eligibility and data will be extracted and entered into RevMan 5.0 in pairs by MZ, JD, HJ and CT. The final review will be written by MZ, AY, JD and HJ.

Editorial group: Cochrane Developmental, Psychosocial and Learning Problems Group.

Publication status and date: Amended to reflect a change in scope (see 'What's new'), published in Issue 3, 2009.

DECLARATIONS OF INTEREST

None known.

This protocol is co-registered within the Campbell Collaboration (www.campbellcollaboration.org). 
(HKD) is similar to ADHD but the criteria are more restrictive. In this review we will use the term ADHD to include Hyperkinetic Disorder, although technically HKD defines a more severe subgroup of ADHD (WHO 1992; APA 1994).

Prevalence estimates for ADHD vary considerably and depend on characteristics of the population, sampling methods and the nature of the assessment (Jadad 1999a; Faraone 2003; Sciutto 2007). Ford et al found that in a UK survey of 10,438 children aged 5 to $15,3.62 \%$ of boys and $0.85 \%$ of girls had a diagnosis of ADHD (Ford 2003). A survey conducted in the USA by the Center for Disease Control and Prevention of 102,353 parents of children aged 4 to 17 found a lifetime childhood diagnosis of $7.8 \%$ (2.5 times as many boys as girls) of whom $4.3 \%$ had received medication as treatment (CDC 2005). A review of 50 prevalence studies (including 20 US and 30 non-US sample populations) suggested that the prevalence is similar in US and non-US populations (Faraone 2003). The authors suggested that the prevalence in certain populations (Iceland, Australia, Italy and Sweden) might be lower, but that studies that directly compare prevalence would be needed to assess this (Faraone 2003). However, the "administrative" prevalence i.e. the frequency of diagnosis in practice, seems to highlight a cultural difference between US and European clinicians as this ratio is estimated to be as high as 20:1 (Santosh 2005).

Aetiology-It is thought that genetic and environmental risk factors interact to cause ADHD rather than operate in isolation (Pliszka 2007; NICE 2008). The genetic contribution to observable phenotypic ADHD traits has been estimated as being up to 76\% (Faraone 2005). No large single gene effect has been identified but the DRD4 and DRD5 genes appear to be involved ( $\mathrm{Li} 2006$ ) and a specific haplotype of the dopamine transporter gene has also been associated with the combined-type ADHD (Asherson 2007). Environmental factors including maternal smoking, alcohol consumption, heroin use in pregnancy, foetal hypoxia, exposure to toxins, injury and zinc deficiency have been suggested as possible influences too (NICE 2008).

Issues regarding diagnosis-The diagnosis of ADHD has stimulated considerable debate and sometimes strong and conflicting views (Jadad 1999b). Inattention, hyperactivity and impulsivity are normal traits in children, especially younger children. There is no reliable test to confirm diagnostic validity so diagnoses depend on clinical judgment. Consensus among experts in the field (Barkley 2002) might exist, but diagnoses may be open to bias. The use of operationalised diagnostic criteria, such as the DSM-IV (APA 1994) or ICD-10 (WHO 1992), may reduce such bias. Professional and national bodies, concerned about the importance of thorough and accurate ADHD diagnoses have issued guidelines to encourage good practice (American Academy of Pediatrics 2001; SIGN 2001; Pliszka 2007; NICE 2008).

Uncertainty regarding diagnostic validity is greatest in preschool children. The predictive validity of DSM-IV diagnoses in 4-6 year olds is clear. Many are likely to continue to meet ADHD diagnostic criteria three years later (Lahey 2004). It has also been argued that an emerging literature supports the validity of preschool ADHD and that non-pharmacological interventions, especially parent training might be particularly valuable in this group (Sonuga-Barke 2006). 
Preschool diagnosis of ADHD is more problematic than in school age children because little data on preschool diagnostic practice exists (Sonuga-Barke 2003a). Factors such as parental expectation might influence the assessment of severity and impairment. Even where particular symptom levels are judged to be out of the normal range for that age, this might be transitory and reflect normal stepwise or non-linear competency development (SonugaBarke 2003a). It is a complex process to establish caseness (meeting the criteria for ADHD) where high levels of inattention and hyperactivity exist in a preschool child. The extent of these traits and their association with impairment in the particular child must be demonstrated in order to meet DSM-IV diagnostic criteria (APA 1994). Because it is uncertain that this can be done in a systematic and precise way it has been suggested that making consistent diagnostic assessments in this age group could be problematic (SonugaBarke 2003a).

Concerns about diagnostic validity led the Preschool ADHD Treatment Study (PATS) to address this issue (Kollins 2006). Kollins et al recently demonstrated that when parent and teacher rated ADHD symptoms are examined, DSM-IV symptoms do not consistently act as meaningful discriminators in identifying ADHD and its subtypes, and that "it may be that other symptoms not routinely assessed in this age group are more saliently associated with ADHD in the preschool years" (Hardy 2007).

Our review requires that trial participants have a valid ADHD diagnosis. In view of the concerns about diagnostic validity of ADHD in preschool children "at risk" of developing ADHD, we decided that this group fell outside the scope of our review. Without definite ADHD diagnoses, uncertainty would exist as to whether behavioural and symptom change with the intervention occurred in children truly at risk of developing ADHD as opposed to those with ODD/CD.

\section{Treatment}

Pharmacological: Over the past decade, a number of systematic reviews on ADHD treatment have been published (Miller 1998; Jadad 1999a; NICE 2000; SIGN 2001; NICE 2006b; NICE 2008). They conclude that stimulant treatments are relatively safe and effective (at least in the short term) at managing the ADHD "core symptoms" i.e. excessive inattention, hyperactivity and impulsivity. Atomoxetine, a nor-adrenergic re-uptake inhibitor has also been found to be effective (Michelson 2001; NICE 2006b; Cheng 2007).

Professional guidelines recommend pharmacological treatments with or without concomitant psychosocial interventions after a comprehensive diagnostic assessment (American Academy of Pediatrics 2001; Taylor 2004; Pliszka 2007; NICE 2008). Whilst consistent treatment with stimulants is associated with maintenance of effectiveness, it is also associated with "mild" suppression of growth (MTA 2004).

Adding psychological interventions to medication has not been demonstrated to improve outcomes significantly (Miller 1998; MTA 1999; Abikoff 2004). However, it has been argued that stimulants do not necessarily lead to long-term benefits (Jensen 2007). Those with more severe symptoms appear to do better with stimulants than behavioural interventions (Santosh 2005) but subgroups of ADHD patients with co-morbid disorders 
might respond differentially to pharmacological and/or psychological treatments (Jensen 2001).

Psychosocial: Children with ADHD/HKD often have multiple problems and co-morbid disorders such as anxiety, depression, oppositional defiant disorder and relationship difficulties, so multi-modal treatment appears to be appropriate (Wells 2000; Taylor 2004; NICE 2008). This might be why in those with less severe symptoms, the smaller advantage of medication over psychological interventions suggests that safety and user preference could lead to behavioural interventions being used as the first choice, adding medication later if necessary (Santosh 2005). Many reasons exist for considering psychosocial interventions in ADHD including: questions of the long-term effectiveness of stimulants; minimal clinical benefits of medication; non-responsiveness to medication; weak responsiveness to medication; intolerance of medication; the clinical needs of younger children; and, ethical and other objections to the use of medication (NICE 2008).

\section{Description of the intervention}

Parent training programmes are psychosocial interventions aimed at training parents in behavioural/cognitive behavioural techniques to enable them to manage their children's challenging or ADHD-related behaviour. They vary in their style and content but are generally manual-based and may involve discussion, the use of video and role play e.g. Webster-Stratton's Incredible Years programme (Webster-Stratton 1998). In addition to the behavioural/cognitive behavioural content fundamental to generic parent training programmes, ADHD-focused parent training often include psycho-educational components about ADHD and how its presence affects a child's functioning and behaviour (Pliszka 2007). They usually comprise 10-20 weekly sessions of 1-2 hours covering a range of areas that include the nature of ADHD, positive reinforcement skills (including attending carefully to appropriate behaviour/play as well as ignoring skills), reward systems, the use of "time out", liaison with teachers and planning ahead to anticipate problems (Pliszka 2007).

\section{How the intervention might work}

The main aim of parent training is "to reduce children's problem behaviour by strengthening parent management skills" (Hartman 2003). Parent training interventions are mainly based on behaviour-management principles that arise from social-learning theory and comprise structured programmes that allow for reliable application by trained professionals (Kazdin 1997; NICE 2006a). They are based on the principle that the essential skills can be learned and practised (Gardner 2004). Parent training interventions in ADHD are generally aimed at both improving the parents' understanding of ADHD as well as increasing their behaviour management skills.

It is hypothesised that deficits in the brain's executive functioning might result in excessive impulsivity whereas an altered motivational state may cause delay aversion (Solanto 2001; Sonuga-Barke 2003b. Hypothetically, to improve ADHD symptoms, parent training could be designed to work on both of these areas: through cognitive work on self-regulation and through motivational interventions focusing on improving delay tolerance (Sonuga-Barke 2003b). 


\section{Why it is important to do this review}

The evidence suggests that parent training can improve behaviour in children with conduct disorder (Kazdin 1997; NICE 2006a), children with behaviour problems (Barlow 1997) and there is also some support for the effectiveness of group-based parenting programmes in improving the emotional and behavioural adjustment in children under the age of three (Barlow 2003). Furthermore, it may be effective in children who have both Conduct Disorder and the ADHD "core symptoms" of inattention, hyperactivity and impulsivity (Hartman 2003).

Problem behaviours are viewed in two broad dimensions: "Externalising" problems include conflict with others, aggression, rule-breaking and oppositional behaviour, whereas "internalising" problems, include problems that might reflect internal stress, such as anxiety, depression, somatic problems and social withdrawal (Sourander 2005).

Longitudinal research suggests that hyperactivity specifically, is a risk factor for future problems (Taylor 1996; Sourander 2005). Taylor followed up hyperactive children over ten years and showed that the presence of pervasive hyperactivity increased the risk of "psychiatric diagnosis, persisting hyperactivity, violence and other conduct problems and, social and peer problems" even when taking into account the existence of comorbid conduct problems (Taylor 1996).

A forty year longitudinal cohort, the National Survey of Health and Development, followed up 3652 adolescents with externalising problems. It showed that those with externalising behaviours are impaired in their health and social development in multiple ways with considerable impact on themselves, their families and society throughout adult life (Coleman 2009). The specific externalising problems that were included in their measure were: disobedience, lying, lack of punctuality, restlessness, truancy, day dreaming in class, and poor response to discipline. Given that restlessness and day dreaming might be proxies for hyperactivity and inattention, it is possible that this cohort reflects young people with ADHD as well as Conduct Disorder/Oppositional Defiant Disorder (CD/ODD).

Other studies that have followed people with ADHD symptoms into adulthood have shown unfavourable outcomes that include educational and occupational impairment in adulthood (Weiss 1985; Mannuzza 1997) and an increased risk of antisocial personality disorder and substance misuse (Mannuzza 1998; Rasmussen 2000) .

The economic cost of anti-social behaviour to society is considerable and cuts across multiple agencies. When costs were applied to data from the Inner London longitudinal study it was shown that those with conduct disorder cost ten times more than those without (Scott 2001). Parent training interventions in conduct disorder are effective (Kazdin 1997; NICE 2006a; Hutchings 2007; Scott 2007) yet until relatively recently they attracted little funding. This was despite the fact that the effect size of these interventions is comparable to that of anti-depressant medication in depressed adults (Scott 2007).

Early conduct problems appear to precede antisocial behaviour in later life. Farrington estimated that it is possible to predict over half of future recidivist delinquents based on their 
aggressive behaviour and a family's ineffective child rearing practices (Farrington 1995). The precise relationship between CD/ODD and ADHD, especially the mechanism of development of antisocial behaviour in children with ADHD, is not, however, fully understood.

In the British Child \& Adolescent Mental Health Survey, 27\% of those with Conduct Disorder and $26 \%$ of those with Oppositional Defiant Disorder also qualified for a diagnosis of ADHD and more than $50 \%$ of those with ADHD had a comorbid behaviour disorder (Ford 2003).

Although co-morbidity exists between ADHD and CD/ODD it is not clear that parent training in children with ADHD, with or without comorbid CD/ODD, is effective at reducing antisocial behaviour or ADHD symptoms. Although NICE recommend parent training as a treatment intervention in ADHD (NICE 2008) it is not clear that it is effective for those with ADHD as this recommendation is based on studies of children under twelve with conduct disorder rather than those with ADHD (NICE 2006a). They nonetheless recommend that clinical services provide all parents or carers of pre-school children a parent-training/education programme as first-line treatment. They also suggest that parents or carers of school age children with "moderate" ADHD symptom impairment be offered parent training programmes, and, that school age children be offered a group treatment programme involving CBT and/or social skills training (NICE 2008).

Given the high comorbidity between ADHD and CD/ODD, it is understandable that NICE have made these recommendations, but the relationship between parent training and ADHD needs to be examined in its own right. The mechanism for development of behaviour problems might be different for the two conditions. We intend to review evidence obtained from randomised controlled trials of parent training interventions in ADHD to establish whether parent training is effective in reducing ADHD symptoms and associated problems in children.

\section{OBJECTIVES}

To determine whether parent-training interventions are effective in reducing ADHD symptoms and associated problems (e.g. disruptive behaviour disorders or child-specific impairments such as learning difficulties) in children and young people aged 5-18 with ADHD, compared to controls with no parent-training interventions.

\section{METHODS}

\section{Criteria for considering studies for this review}

\section{Types of studies}

- Randomised or quasi randomised (where sequence generation is for example by birth date or alternate allocation) controlled trials

- Studies must contain at least one measure of ADHD-related behaviour (i.e., studies reporting only on parental stress, satisfaction or willingness to medicate, will be excluded) 
Types of participants-Children and young people aged 5 to 18 years in whom the main problem is Attention Deficit Hyperactivity Disorder diagnosed using operationalised diagnostic criteria of the DSM-IV or its earlier versions (since the ICD-10 definition of hyperkinetic disorder is narrower than the DSM-IV definition of ADHD, ICD-10 diagnoses of hyperkinetic disorder are also considered to be acceptable). The diagnoses should be clinical diagnoses by specialists with or without the use of semi-structured or structured interview instruments. Acceptable diagnoses include:

- Attention-Deficit/Hyperactivity Disorder (DSM III-R, DSM-IV) (APA 1987; APA 1994).

- Attention Deficit Disorder (DSM III) (APA 1980).

- Hyperkinetic Disorder (ICD-9, 1CD-10) (WHO 1977; WHO 1992).

Types of interventions-Parent-training interventions where the intervention was designed to train parents in behavioural and/or cognitive behavioural interventions to improve the management of their child's ADHD-related difficulties. Parent-training may be:

- Group-based interventions.

- Interventions for individual parents, or for a couple.

- The combination of individual/couple and group interventions.

- Trials in which the parents are the main mediators of the parent training intervention but in which a teacher component, by teacher/s trained in behavioural management similar to the parent-training component, takes place.

Trials will be excluded where direct interventions with the children are used. This is to separate out the effect of parent training and the effect of the direct behavioural intervention with the child and eliminate the possibility of interaction between them.

However, trials in which drug treatments are used alongside parent-training interventions will be included (i.e. parent training plus medication versus medication alone) and subgroup analysis of trials in which drug treatments are used will be undertaken.

The following comparisons will be made where data are available:

- Parent training versus a no treatment or wait list control.

- Parent training versus routine care (treatment as usual).

- Parent training versus unstructured group parent support meetings.

\section{Types of outcome measures}

\section{Primary outcomes}

- Change in the child's ADHD-symptom-related behaviour in home setting (e.g. Conner's or SNAP questionnaires) (Conners 1998a).

- Change in the child's ADHD-symptom-related behaviour in school setting (e.g. Conner's Teacher Rating Scale) (Conners 1998b). 
- Changes in the child's general behaviour (e.g. Achenbach Child Behaviour Checklist) (Achenbach 1991).

- Academic achievement measured through school test result.

\section{Secondary outcomes}

- *Adverse events.

- ${ }^{*}$ Changes in parenting skills (e.g. The Parenting Clinical Observation Schedule (Hill 2008)).

- *Parental stress (e.g. the Parenting Stress Index (Abidin 1995)).

- Parental understanding of ADHD (e.g. ADHD Knowledge \& Opinion Scale (Rostain 1993)),

Outcome measures may be reports by clinician, parent, teacher or trained investigator. Instruments used must be published in a peer-reviewed journal and validated in the population i.e. tested for validity in children/young people with ADHD and shown to measure the change that they set out to measure.

Outcomes marked by asterisks indicate outcomes planned for inclusion within 'Summary of findings' tables at review stage (Schünemann 2008).

\section{Search methods for identification of studies}

Electronic searches-The following electronic databases will be searched:

- Cochrane Central Register of Controlled Trials

- MEDLINE

- EMBASE

- CINAHL

- PsycINFO

- Dissertation Abstracts International, searched through Dissertation Express

- ClinicalTrials.gov

The full search strategy that will be used for CENTRAL appears Appendix 1. It will be modified, where necessary, for the other databases listed. Appropriate trials filters will be added to each strategy where necessary.

Searching other resources-References in previous reviews and studies will be checked. Authors and known experts will be contacted to identify any additional or unpublished data.

\section{Data collection and analysis}

Selection of studies-Initial screening of abstracts and titles from the search to identify potential trials for inclusion will be undertaken independently, by two researchers working 
in pairs (MZ, HJ \& JD). who will then independently assess and select studies for inclusion. If disagreement occurs consensus will be reached through discussion and by consultation with the Coordinating Editor of the CDPLPG (Professor Geraldine Macdonald). A flow chart of the process of trial selection will be made in accordance with the QUORUM statement (Moher 1999).

\section{Data extraction and management}

Data extraction-Three authors (MZ, CT and HJ) will extract data independently using data-extraction sheets that had been previously piloted to check for reliability in extracting the relevant data. Citations will be stored and organised in ProCite bibliographic software.

Data collection-When more than two treatment arms were included in the same trial, all arms will be described.

The following data will be collected for all trial arms:

1. Descriptive data, including participant demographics (age, gender, baseline measures of school achievement, social and economic status);

2. Intervention characteristics (including delivery, duration, and within-intervention variability);

3. Other interventions received (including delivery and duration);

4. Outcome measures listed above.

\section{Assessment of risk of bias in included studies}

Risk of bias within each included study will by assessed independently by at least two review authors according to the Cochrane Collaboration Handbook (Higgins 2008a; Higgins 2008b). Review authors will independently assess the risk of bias within each included study based on the following six domains with ratings of 'Yes' (low risk of bias); 'No' (high risk of bias) and 'Unclear' (uncertain risk of bias):

Sequence generation-Description: the method used to generate the allocation sequence will be described in detail so as to assess whether it should have produced comparable groups; review authors' judgment: was the allocation concealment sequence adequately generated?

Ratings: 'Yes' (low risk of bias); 'No' (high risk of bias) and 'Unclear' (uncertain risk of bias)

Allocation concealment-Description: the method used to conceal allocation sequence will be described in sufficient detail to assess whether intervention schedules could have been foreseen in advance of, or during, recruitment; review authors' judgment: was allocation adequately concealed?

Ratings: 'Yes' (low risk of bias); 'No' (high risk of bias) and 'Unclear' (uncertain risk of bias) 
Blinding-Description: any measures used to blind participants, personnel and outcome assessors will be described so as to assess knowledge of any group as to which intervention a given participant might have received; review authors' judgment: was knowledge of the allocated intervention adequately prevented during the study?

Ratings: 'Yes' (low risk of bias); 'No' (high risk of bias) and 'Unclear' (uncertain risk of bias)

Incomplete outcome data-Description: If studies do not report intention-to-treat analyses, attempts will be made to obtain missing data by contacting the study authors. Data on attrition and exclusions will be extracted and reported as well the numbers involved (compared with total randomised), reasons for attrition/exclusion where reported or obtained from investigators, and any re-inclusions in analyses performed by review authors; review authors' judgment: were incomplete data dealt with adequately by the reviewers? (See also 'Dealing with missing data', below).

Ratings: 'Yes' (low risk of bias); 'No' (high risk of bias) and 'Unclear' (uncertain risk of bias)

Selective outcome reporting-Description: attempts will be made to assess the possibility of selective outcome reporting by investigators; The review authors' judgment will be based on the question: Are reports of the study free of suggestion of selective outcome reporting?

Ratings: 'Yes' (low risk of bias); 'No' (high risk of bias) and 'Unclear' (uncertain risk of bias)

Validity and reliability of outcome measures used-Description: Were the outcome measures standardised and validated for the population?

Ratings: 'Yes' (low risk of bias); 'No' (high risk of bias) and 'Unclear' (uncertain risk of bias)

Other sources of bias-Description: Was the study apparently free of other problems that could put it at a high risk of bias?

Ratings: 'Yes' (low risk of bias); 'No' (high risk of bias) and 'Unclear' (uncertain risk of bias)

\section{Measures of treatment effect}

Binary data-For dichotomous (binary) data, the odds ratio with a 95\% confidence interval will be used to summarise results within each study. The odds ratio is chosen because it has statistical advantages relating to its sampling distribution and its suitability for modelling, and because it is a relative measure and so can be used to combine studies.

Categorical data-Where results are reported in short ordinal scales, the methods of Whitehead and Jones will be used to produce a single odds ratio from each trial (Whitehead 
1994). If sufficient detail is not available we will consider analysing such scales as continuous data, after investigating skew and appropriateness.

Continuous data-If continuous outcomes are measured identically across studies, an overall weighted mean difference (WMD) and 95\% CI may be calculated. If the same continuous outcome is measured differently across studies, an overall standardised mean difference (SMD) and 95\% CI may be calculated (Higgins 2005). SMDs will be calculated using Hedges $g$.

\section{Unit of analysis issues}

Cluster-randomised trials-Where trials have used clustered randomisation, we anticipate that study investigators would have presented their results after appropriately controlling for clustering effects (robust standard errors or hierarchical linear models). If it is unclear whether a cluster-randomised trial has used appropriate controls for clustering, the study investigators will be contacted for further information. Where appropriate controls were not used, individual participant data will be requested and re-analysed using multilevel models which control for clustering. Following this, effect sizes and standard errors will be meta-analysed in RevMan using the generic inverse method (RevMan 2008). If appropriate controls were not used and individual participant data is not available, statistical guidance will be sought from the Cochrane Method Group and external experts as to which method to apply to the published results in attempt to control for clustering. If there is insufficient information to control for clustering, outcome data will be entered into RevMan using individuals as the units of analysis, and then sensitivity analysis will be used to assess the potential biasing effects of inadequately controlled clustered trials (Donner 2001).

\section{Dealing with missing data}

When necessary, the corresponding author will be contacted to supply any unreported data (e.g., group means and standard deviations (SDs), details of dropouts, and details of interventions received by the control group). Other authors will be contacted if necessary. If a study reports outcomes only for participants completing the trial or only for participants who followed the protocol, authors will be contacted and asked to provide additional information to permit an intention-to-treat analyses.

\section{Assessment of heterogeneity}

We will assess the extent of heterogeneity using the three methods suggested by the Cochrane Handbook (Deeks 2008): visual inspection of forest plots, the chi square statistic (increasing the level of significance to 0.10 to avoid underestimating heterogeneity) and using Higgins's $\mathrm{I}^{2}$ statistic designed to assess the impact of heterogeneity on the metaanalysis. It describes the "percentage of the variability in effect estimates that is due to heterogeneity rather than sampling error (chance)" (Higgins 2002; Higgins 2003). However, it is advised that the thresholds of the $\mathrm{I}^{2}$ statistic might be misleading and the following guide is offered:

- $0-40 \%$ : might not be important

- 30\%-60\%: may represent moderate heterogeneity 
- $50 \%$ - 90\%:may represent substantial heterogeneity

- 75\%-100\%: considerable heterogeneity

We will bear in mind that the 'importance of the observed value of $\mathrm{I}^{2}$ depends on (i) magnitude and direction of effects and (ii) strength of evidence for heterogeneity (e.g. P value from the chi-squared test, or a confidence interval for $\mathrm{I}^{2}$ )' (Higgins 2008a).

\section{Assessment of reporting biases}

To investigate the possibility of reporting biases, including publication bias, funnel plots will be drawn (Egger 1997; Deeks 2005; Sterne 2001). In the event of asymmetry, the reviewers will seek input from methodologists, including the Cochrane and Campbell Collaboration Methods Groups, on appropriate analyses, given concerns raised in Chapter 10.4.2 of the Cochrane Handbook (Sterne 2008).

We plan also to address the issue of selective outcome reporting by checking against the included studies' protocols if possible (within trial registries, conference proceedings, etc.), and by internal evidence within the published studies.

\section{Data synthesis}

Outcome data-We intend to undertake a quantitative synthesis of data. Where sufficient clinical and methodological homogeneity exists between trials, results will be pooled. We are aware of the dangers of interpreting findings of single or even multiple studies in the absence of meta-analysis. Where meta-analysis is not possible, for example, where outcomes measure different domains such as "ADHD core symptoms" and "educational achievement", we will provide reasons and report investigators' findings narratively.

RevMan 5.0 (RevMan 2008) will be used to perform the following calculations. All overall effects will be calculated using inverse variance methods. We will undertake both fixed and random effects analyses to describe our findings. It is likely that the studies will yield heterogeneous data, because of diagnostic variability in ADHD and differences in parent training models used by different researchers.

Types of analyses-Studies in which participants are analysed as members of the groups to which they were originally assigned (intention-to-treat analysis), studies that include only those participants who were willing or able to provide data (available-case analysis), and studies that analyse participants who adhered to the study's design (per-protocol analysis; Higgins 2005) will be analysed separately. Studies in which the reasons for excluding participants from analyses can not be determined from relevant reports or through contact with the authors will be considered with per-protocol analyses.

Multiple arms-All eligible outcome measures for all trial arms will be reported in the review.

If two or more eligible intervention groups are compared to an eligible control, review authors may consider combining data for interventions provided each meets all inclusion 
criteria and do not (as per exclusion criteria above) involve unacceptable adjunct treatments e.g. direct work with children.

If a single eligible intervention group is compared to multiple eligible control groups, 'notreatment' controls will be chosen over other groups for comparison and inclusion in metaanalyses (Lipsey 2001). For studies that do not have no-treatment condition, the most appropriate eligible alternative will be chosen (see list of comparisons, above).

Multiple measures-When a single study provides multiple measures of the same outcome (e.g. two measures are used to ADHD symptoms) we will average the effects from the outcomes to arrive at a single effect for use in the meta-analysis.

\section{Subgroup analysis and investigation of heterogeneity}

Large numbers of subgroups may lead to misleading conclusions and are best kept to a minimum (Yusuf 1991; Oxman 1992). If possible, this review will include separate effect estimates for the following subgroups:

- children receiving concomitant drug treatment;

- children with disruptive behaviour disorders where ADHD is specified as a subgroup;

- parent-training programmes that are group based versus individual or couple based programmes;

- parent-training programmes that involve a teacher component.

\section{Sensitivity analysis}

Sensitivity analyses will be carried out:

- to analyse the differential impact of true versus 'quasi' randomised studies;

- where loss to follow-up exceeds $20 \%$;

- Sensitivity analysis will be carried out to compare studies in which fidelity testing was undertaken compared to those where it was not done.

Qualitative data-Qualitative data from included studies may be included to better understand the delivery of interventions, uptake by participants, and context.

\section{Acknowledgments}

We would like to thank persons formerly on the authorline of this protocol who made contributions at early stages (Carol Joughin, Sima Pindoria (UK)). Thanks also to members of the original steering group at the Systematic Reviews Training Unit, Institute of Child Health, University College London (UK), especially Stuart Logan.

Thanks also to Geraldine Macdonald (Coordinating Editor, CDPLPG) for hard work and support of this project; to Jo Abbott (TSC of the CDPLPG) for searches; and to anonymous statistical editors within the Cochrane and Campbell Collaborations. We are also grateful to Krystyna Kowalski (SFI Campbell, Denmark) for patience and support over several years and to Cathy Bennett (Systematic Research Ltd., UK) for continuing assistance and attention to detail. 
We would also like to thank those who helped us in obtaining potential studies for the review to come, including: Julie Millener of the CDPLPG editorial base, Yanina Sguassero (CDPLPG author, Argentina) and Jutta Stoffers (CDPLPG author, Germany). Thanks also to Angela Huertas-Ceballos (CDPLPG author, UK), Toby Lasserson of the Cochrane Airways Group (UK), and Danielle Ouwejan of the University of Bristol (UK) who provided assistance with translations of potential studies from Spanish, German and Dutch, respectively.

\section{SOURCES OF SUPPORT}

Internal sources

- University of Bristol, UK.

External sources

- PPP Healthcare Medical Trust "Mid-Career Awards" grant, UK.

- $\quad$ SFI Campbell, The Danish National Centre for Social Research, Denmark.

\section{Appendix 1. CENTRAL search strategy}

1. MeSH descriptor Psychotherapy explode all trees

2. MeSH descriptor Family Relations explode all trees

3. (parent* or famil* or father* or mother* or paternal* or maternal* or couple* or marital*)

4. (psycho* therap*)

5. (behavio* near therap*)

6. behavio* near intervention*

7. behavio* near treatment*

8. multimodal* or multi-modal*

9. (mta)

10. (parent* near program*)

11. parent* near train*

12. parent* near educat*

13. parent* near promot*

14. parent-train*

15. parent-educat*

16. parent-promot*

17. parent* near therap*

18. (\#1 OR \#2 OR \#3 OR \#4 OR \#5 OR \#6 OR \#7 OR \#8 OR \#9 OR \#10 OR \#11 OR \#12 OR \#13 OR \#14 OR \#15 OR \#16 OR \#17)

19. MeSH descriptor Attention Deficit and Disruptive Behavior Disorders explode all trees

20. attention near deficit 
21. child* near attention

22. child* near inattention

23. child* near impulsiv*

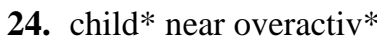

25. hyperkin*

26. hyper near activ*

27. over near activ*

28. hyper near kin*

29. hyperactiv*

30. disruptiv*

31. adhd or addh

32. ad next hd

33. minimal next brain

34. brain and dysfunction

35. (\#19 OR \#20 OR \#21 OR \#22 OR \#23 OR \#24 OR \#25 OR \#26 OR \#27 OR \#28 OR \#29 OR \#30 OR \#31 OR \#32 OR \#33 OR \#34)

36. (\#18 AND\#35)

\section{WHAT'S NEW}

\begin{tabular}{lll}
\hline Date & Event & Description \\
\hline 1 May 2009 & $\begin{array}{l}\text { New citation required and } \\
\text { major changes }\end{array}$ & $\begin{array}{l}\text { This protocol has been substantially updated since first publication in } \\
\text { 2001 and has been co-registered within the Campbell Collaboration }\end{array}$ \\
\hline
\end{tabular}

\section{HISTORY}

Protocol first published: Issue 2, 2001

\begin{tabular}{lll}
\hline Date & Event & Description \\
\hline 9 September 2008 & Amended & Converted to new review format. \\
\hline 9 September 2008 & Amended & Hannah Jones added to authorline \\
\hline 4 July 2007 & Amended & $\begin{array}{l}\text { Camilla Thorgaard added to authorline; Sima Pindoria and } \\
\text { Carol Joughin removed }\end{array}$ \\
\hline 20 April 2005 & $\begin{array}{l}\text { New citation required and } \\
\text { conclusions have changed }\end{array}$ & Substantive amendment \\
\hline
\end{tabular}




\section{Additional references}

Abidin 1995 . Abidin, RR. Parenting Stress Index (PSI). Psychological Assessment Resources, Inc; Lutz, Florida USA: 1995.

Abikoff 2004 . Abikoff H, Hechtman L, Klein RG, Weiss G, Fleiss K, Etcovitch J, et al. Symptomatic Improvement in Children with ADHDH Treated With Long-Term Methylphenidate and Multimodal Psychosocial Treatment. Journal of the American Academy of Child and Adolescent Psychiatry. 2004; 43(7):802-11. [PubMed: 15213581]

Achenbach 1991 . Achenbach, TM. Manual for the Child Behavior Checklist /4-18 and 1991 profile. University of Vermont Department of Psychiatry; Burlington, VT: 1991.

American Academy of Pediatrics 2001 . American Academy of Pediatrics. Clinical Practice Guideline: Treatment of the School-Aged Child With Attention Deficit Hyperactivity Disorder. Pediatrics. 2001; 108(4):1033-44. [PubMed: 11581465]

APA 1980 . American Psychiatric Association. Diagnostic and Statistical Manual of Mental Disorders, Third Edition (DSM III). American Psychiatric Association; Washington DC: 1980.

APA 1987 . American Psychiatric Association. Diagnostic and Statistical Manual of Mental Disorders, 3rd edition-Revised (DSM-III-R). American Psychiatric Association; Washington, DC: 1987.

APA 1994 . American Psychiatric Association. Diagnostic and Statistical Manual of Mental Disorders, 4th edition (DSM-IV). American Psychiatric Association; Washington DC: 1994.

Asherson 2007 . Asherson P, Brookes K, Franke B, Chen W, Gill M, Ebstein RP, et al. Confirmation that a Specific Haplotype of the Dopamine Transporter Gene Is Associated With Combined-Type ADHD. American Journal of Psychiatry. 2007; 164:674-7. [PubMed: 17403983]

Barkley 2002 . Barkley RA. International Consensus Statement on ADHD. Journal of the American Academy of Child \& Adolescent Psychiatry. 2002; 41(12):1389. [PubMed: 12447019]

Barlow 1997 . Barlow, J. Systematic review of the effectiveness of parent-training programmes in improving the behaviour of 3-7 year old children: Health Services Research Unit Report. HSRU; Oxford: 1997.

Barlow 2003 . Barlow J, Parsons J. Group-based parent-training programmes for improving emotional and behavioural adjustment in 0-3 year old children. Cochrane Database of Systematic Reviews. 2003; (2) [Art. No.: CD003680. DOI: 10.1002/14651858.CD003680].

CDC 2005 . Center for Disease Control. Mental Health in the United States: Prevalence of Diagnosis and Medication Treatment for Attention-Deficit/Hyperactivity Disorder --- United States, 2003. Sep 2; 2005 54(34):842-47. MMWR Weekly. http://www.cdc.gov/mmwr/preview/mmwrhtml/ mm5434a2.htm

Cheng 2007 . Cheng JY, Chen RY, Ko JS, Ng EM. Efficacy and safety of atomoxetine for attentiondeficit/hyperactivity disorder in children and adolescents-meta-analysis and meta-regression analysis. Psychopharmacology. 2007; 194(2):197-209. [PubMed: 17572882]

Coleman 2009 . Coleman JM, Abbott RA, Maughan B, Kuh D, Croudace TJ, Jones PB. Outcomes of conduct problems in adolescence: 40 year follow-up of national cohort. BMJ. 2009; 338(a2981) epub.

Conners 1998a . Conners CK, Sitarenios G, Parker JDA, Epstein JN. The revised Conners' Parent Rating Scale (CPRS-R): Factor structure, reliability, and criterion validity. Journal of Abnormal Child Psychology. 1998; 26(4):257-68. [PubMed: 9700518]

Conners 1998b . Conners CK, Sitarenios G, Parker JD, Epstein JN. Revision and restandardization of the Conners' Teacher Rating Scale (CTRS-R) Factor structure reliability, and criterion validity. Journal of Abnormal Child Psychology. 1997; 26:279-91. [PubMed: 9700520]

Deeks 2005 . Deeks J, Macaskill P, Irwig L. The performance of tests of publication bias and other sample size effects in systematic reviews of diagnostic test accuracy was assessed. Journal of Clinical Epidemiology. 2005; 58(9):882-93. [PubMed: 16085191]

Deeks 2008 . Deeks, JJ.; Higgins, JPT.; Altman, DG. Section 9.5.2. Analysing data and undertaking meta-analyses. In: Higgins, JPT.; Green, S., editors. Cochrane Handbook for Systematic Reviews of Interventions version 5.01 (updated Sept 2008). Cochrane Collaboration; 2008. 
Donner 2001 . Donner A, Piaggio G, Villar J. Statistical methods for the meta-analysis of cluster randomization trials. Statistical Methods in Medical Research. 2001; 10(5):325-38. [PubMed: 11697225]

Egger 1997 . Egger M, Davey-Smith G, Schneider M, Minder C. Bias in meta-analysis detected by a simple, graphic test. British Medical Journal. 1997; 315(7):629-34. [PubMed: 9310563]

Faraone 2003 . Faraone S, Sergeant J, Gillberg C, Biederman J. The worldwide prevalence of ADHD:is it an American condition? World Psychiatry. 2003; 2(2):104-13.http:// www.pubmedcentral.nih.gov/picrender.fcgi?artid=1525089\&blobtype=pdf [PubMed: 16946911]

Faraone 2005 . Faraone SV, Perlis RH, Doyle AE, Smoller JW, Goralnick JJ, Holmgren MA, Sklar P. Molecular genetics of attention-deficit/hyperactivity disorder. Biological Psychiatry. 2005; 57(11):1313-1323. [PubMed: 15950004]

Farrington 1995 . Farrington DP. The development of offending and antisocial behaviour from childhood: key findings from the Cambridge study in delinquent development. Journal of Child Psychology and Psychiatry and Allied Disciplines. 1995; 36:929-64.

Ford 2003 . Ford T, Goodman R, Meltzer H. The British Child and Adolescent Mental Health Survey 1999: the prevalence of DSM-IV disorders. Journal of the American Academy of Child and Adolescent Psychiatry. 2003; 42:1203-11. [PubMed: 14560170]

Gardner 2004 . Gardner, F.; Hutchings, J.; Lane, E. Three to eight years: risk and protective factors; effective interventions. In: Sutton, C.; Utting, D.; Farrington, D., editors. Support from the start: working with young children and their families to reduce the risks of crime and anti-social behaviour (research report 524). Department for Education and Skills; Norwich: 2004. p. 43-57.

Hardy 2007 . Hardy KK, Kollins SH, Murray DW, Riddle MA, Greenhill L, Cunningham C, et al. Factor Structure of Parent- and Teacher-Rated Attention-Deficit/Hyperactivity DisorderSymptoms in the Preschoolers with Attention-Deficit/Hyperactivity Disorder Treatment Study (PATS). Journal of Child and Adolescent Psychopharmacology. 2007; 17(5):621-33. [PubMed: 17979582]

Hartman 2003 . Hartman R, Stage S, Webster-Stratton C. A growth curve analysis of parent training outcomes: Examining the influence of child risk factors (inattention, impulsivity, and hyperactivity problems), parental and family risk factors. Journal of Child Psychology and Psychiatry and Allied Disciplines. 2003; 44(3):388-98.

Higgins 2002 . Higgins JPT, Thompson SG. Quantifying heterogeneity in a meta-analysis. Statistics in Medicine. 2002; 21:1539-58. [PubMed: 12111919]

Higgins 2003 . Higgins J, Thompson SG, Deeks J, Altman D. Measuring inconsistency in metaanalyses. BMJ Clinical Research. 2003; 327(7414):557-60.

Higgins 2005 . Higgins, JPT.; Green, S., editors. The Cochrane Library. John Wiley \& Sons, Ltd; Chichester, UK: 2005. Cochrane Handbook for Systematic Reviews of Interventions 4.2.5 [updated May 2005].

Higgins 2008a . Higgins, JPT.; Altman, DG. Cochrane Handbook for Systematic Reviews of Interventions version 5.01 (updated Sept 2008). Higgins, JPT.; Green, S., editors. The Cochrane Collaboration; 2008.

Higgins 2008b . Higgins, JPT.; Altman, DG. Section 8. Assessing risk of bias in included studies. In: Higgins, JPT.; Green, S., editors. Cochrane Handbook for Systematic Reviews of Interventions version 5.01 (updated Sept 2008). The Cochrane Collaboration; 2008.

Hill 2008 . Hill C, Maskowitz K, Danis B, Wakschlag L. Validation of a clinically sensitive, observational coding system for parenting behaviors: The Parenting Clinical Observation Schedule. Parenting: Science \& Practice. 2008; 8(2):153-85.

Hutchings 2007 . Hutchings J, Bywater T, Daley D, Gardner F, Whitaker C, Jones K, Eames C, Edwards R. Parenting intervention in Sure Start services for children at risk of developing conduct disorder: pragmatic randomised controlled trial. BMJ. 2007; 334(678) Epub (no page numbers given).

Jadad 1999b . Jadad AR, Brooker L, Gauld M, Kakuma R, Boyle M, Cunningham CE, et al. The treatment of attention-deficit/hyperactivity disorder: an annotated bibliography and critical appraisal of published systematic reviews and meta-analyses. Canadian Journal of Psychiatry. $1999 ; 44: 1025-35$. 
Jadad 1999a Jadad, AR.; Boyle, MH.; Cunningham, C.; Kim, M.; Scachar, R. Treatment of attention-deficit/hyperactivity disorder. Evidence Report/Technology Assessment no 11 (prepared for McMaster University under contract no. 290-97-0017). Agency for Healthcare Research and Quality; Rockville, Maryland, USA: 1999. AHRQ Publication No. 00-E005Vol. Summary Publication No.99-E017, Evidence Report/Technology Assessment

Jensen 2001 . Jensen PS, Hinshaw SP, Kraemer HC, Lenora N, Newcorn JH, Abikoff HB, et al. ADHD Comorbidity Findings From the MTA Study: Comparing Comorbid Subgroups. Journal of the American Academy of Child and Adolescent Psychiatry. 2001; 40(2):147-158. [PubMed: 11211363]

Jensen 2007 . Jensen PS, Arnold LE, Swanson JM, Vitiello B, Abikoff HB, Greenhill LL, et al. 3Year Follow-up of the NIMH MTA Study. Journal of the American Academy of Child and Adolescent Psychiatry. 2007; 46(8):989-1002. [PubMed: 17667478]

Kazdin 1997 . Kazdin A. Parent management training: evidence, outcomes, and issues. Journal of the American Academy of Child \& Adolescent Psychiatry. 1997; 36(10):1349-56. [PubMed: 9334547]

Kollins 2006 . Kollins S, Greenhill L, Swanson J, Wigal S, Abikoff H, McCracken J, et al. Rationale, Design, and Methods of the Preschool ADHD Treatment Study (PATS). Journal of the American Academy of Child \& Adolescent Psychiatry. 2006; 45(11):1275-83. [PubMed: 17023869]

Lahey 2004 . Lahey B, Pelham W, Loney J, Kipp H, Ehrhardt A, Lee SS, et al. Three-Year Predictive Validity of DSM-IVAttention Deficit Hyperactivity Disorderin Children Diagnosed at 4-6 Years of Age. American Journal of Psychiatry. 2004; 161(11):2014-20. [PubMed: 15514401]

Li 2006 . Li D, Sham PC, Owen MJ, He L. Meta-analysis shows significant association between dopamine system genes and attention deficit hyperactivity disorder. Human Molecular Genetics. 2006; 15:2276-84. [PubMed: 16774975]

Lipsey 2001 . Lipsey, MW.; Wilson, DB. Practical Meta-analysis. Thousand Oaks; Sage, CA: 2001.

Mannuzza 1997 . Mannuzza S, Klein RG, Bessler A, Malloy P, Hynes ME. Educational and occupational outcome of hyperactive boys grown up. Journal of the American Academy of Child \& Adolescent Psychiatry. 1997; 36(9):1222-27. [PubMed: 9291723]

Mannuzza 1998 . Mannuzza, Salvatore; Rachel, G.; Klein, Abrah Bessler; Malloy, Patricia; LaPadula, Maria. Adult Psychiatric Status of Hyperactive Boys Grown Up. American Journal of Psychiatry. 1998; 155:493-498. [PubMed: 9545994]

Michelson 2001 . Michelson D, Faries D, Wernicke J, Kelsey D, Kendrick K, Sallee F, et al. Atomoxetine in the treatment of children and adolescents with attention-deficit/hyperactivity disorder: a randomized, placebo-controlled, dose-response study. Pediatrics. 2001; 108(5):E83. [PubMed: 11694667]

Miller 1998 . Miller, A.; Lee, S.; Raina, P.; Klassen, A.; Zupancic, J.; Olsen, L. A review of therapies for attention-deficit/hyperactivity disorder. Canadian Coordinating Office for Health Technology Assessment (CCOHTA); Ottawa: Dec. 1998

Moher 1999 . Moher D, Cook D, Eastwood S, Olkin I, Rennie D, Stroup D. Improving the quality of reports of meta-analyses of randomised controlled trials: the QUOROM statement. Lancet. 1999; 354:1896-900. [PubMed: 10584742]

MTA 1999 . The MTA Cooperative Group. A 14-month randomized clinical trial of treatment strategies for attention-deficit/hyperactivity disorder. Archives of General Psychiatry. 1989; 56(12):1073-86.

MTA 2004 . MTA Cooperative Group. National Institute of Mental Health Multimodal Treatment Study of ADHD Follow-up: Changes in Effectiveness and Growth after the End of Treatment. Pediatrics. 2004; 113(4):762-9. [PubMed: 15060225]

NICE 2000 . Lord J, Paisley S. The clinical effectiveness and cost-effectiveness of methylphenidate for hyperactivity in childhood. Technical Appraisal 13, National Institute for Clinical Excellence August 2000, issue Version 2. http://www.nice.org.uk/nicemedia/pdf/Methylphenidatereport.pdf 
NICE 2006a . National Institute for Health and Clinical Excellence \& Social Care Institute for Excellence. Parent-training/education programmes in the management of children with conduct disorders. NICE Technology Appraisal 102; Jul. 2006

NICE 2006b . National Institute for Health and Clinical Excellence. [accessed 14 February 2009] Methylphenidate, atomoxetine and dexamfetamine for the treatment of attention deficit hyperactivity disorder in children and adolescents. 2006. Available online at: www.nice.org.uk/ TA98

NICE 2008 . National Institute for Health and Clinical Excellence. Attention Deficit Hyperactivity Disorder: Diagnosis and management of ADHD in children, young people and adults. Jan. 2008 p. 1-591.accessed 9 September 2008

Oxman 1992 . Oxman A, Guyatt G. A consumer's guide to subgroup analyses. Annals of Internal Medicine. 1992; 116(1):78-84. [PubMed: 1530753]

Pliszka 2007 . Pliszka S, Bernet W, Bukstein O, Walter H, Arnold V, Beitchman J, et al. Practice Parameters for the Assessment and Treatment of Children and Adolescents with Attention Deficit Hyperactivity Disorder. Journal of the American Academy of Child and Adolescent Psychiatry. 2007; 46(7):894-921. [PubMed: 17581453]

Rasmussen 2000 . Rasmussen P, Gillberg C. Natural outcome of ADHD with developmental coordination disorder at age 22 years: A controlled, longitudinal, community-based study. Journal of the American Academy of Child \& Adolescent Psychiatry. 2000; 39(11):1424-31. [PubMed: 11068898]

RevMan 2008 . The Cochrane Collaboration. Review Manager (RevMan) [Computer program]. Version 5.0. The Cochrane Collaboration; Nordic Cochrane Center, Copenhagen: 2008.

Rostain 1993 . Rostain AL, Power TJ, Atkins MS. Assessing parents' willingness to pursue treatment for children with attention-deficit hyperactivity disorder. Journal of the American Academy of Child \& Adolescent Psychiatry. 1993; 32(1):175-81. [PubMed: 8428869]

Santosh 2005 . Santosh PJ, Taylor E, Swanson J, Wigal T, Chuang S, Davies M, et al. Refining the diagnoses of inattention and overactivity syndromes: A reanalysis of the Multimodal Treatment study of attention deficit hyperactivity disorder (ADHD) based on ICD-10 criteria for hyperkinetic disorder. Clinical Neuroscience Research. 2005; 5:295-306.

Schünemann 2008 . Schünemann, HJ.; Oxman, AD.; Higgins, SPT.; Vist, GE.; Glasziou, P.; Guyatt, GH. Chapter 11: Presenting results and 'Summary of findings' tables. In: Higgins, JPT.; Green, S., editors. Cochrane Handbook for Systematic Reviews of Interventions. The Cochrane Collaboration; 2008. Available from www.cochrane-handbook.org

Sciutto 2007 . Scuitto M, Eisenberg M. Evaluating the Evidence For and Against the Overdiagnosis of ADHD. Journal of Attention Disorders. 2007; 11(2):106-113. [PubMed: 17709814]

Scott 2001 . Scott S, Knapp M, Henderson J, Maughan M. Financial cost of social exclusion: follow up study of antisocial children into adulthood. BMJ. 2001; 323:1-5. [PubMed: 11440920]

Scott 2007 . Scott S. Conduct disorders in children. BMJ. 2007; 334:646. [PubMed: 17395905]

SIGN 2001 . Scottish Intercollegiate Guidelines Network. [accessed 26 February 2009] Attention Deficit and Hyperkinetic Disorders in Children and Young People: A National Guideline. 2001. http://www.sign.ac.uk/pdf/sign52.pdfhttp://www.sign.ac.uk/pdf/sign52.pdf

Solanto 2001 . Solanto M, Abikoff H, Sonuga-Barke E, Schachar R, Logan GD, Wigal T, et al. The ecological validity of delay aversion and response inhibition as measures of impulsivity in ADHD: a supplement to the NIMH multi-modal treatment study of AD/HD. Journal of Abnormal Child Psychology. 2001; 29:215-28. [PubMed: 11411784]

Sonuga-Barke 2003a . Sonuga-Barke E, Daley D, Thompson M, Swanson J. Preschool ADHD: Exploring uncertainties in diagnostic validity and utility, and treatment efficacy and safety. Expert Review Neurotherapeutics. 2003; 3(4):465-76.

Sonuga-Barke 2003b . Sonuga-Barke E, Dalen L, Remington B. Do Executive Deficits and Delay Aversion MakeIndependent Contributions to PreschoolAttention-Deficit/Hyperactivity Disorder Symptoms? Journal of the American Academy of Child \& Adolescent Psychiatrists. 2003; 42(11):1335-42. 
Sonuga-Barke 2006 . Sonuga-Barke E, Thompson M, Abkkoff Klein R, Brotman LM. Nonpharmacological Interventions for Preschoolers With ADHD. Infants \& Young Children. 2006; 19(2):142-153.

Sourander 2005 . Sourander A, Helstelä L. Childhood predictors of externalizing and internalizing problems in adolescence: A prospective follow-up study from age 8 to16. European Child \& Adolescent Psychiatry. 2005; 14(8):415-23. [PubMed: 16341497]

Sterne 2001 . Sterne J, Egger M. Funnel plots for detecting bias in meta-analysis: guidelines on choice of axis. Journal of Clinical Epidemiology. 2001; 54(10):1046-55. [PubMed: 11576817]

Sterne 2008 . Sterne, JAC.; Egger, M.; Moher D on behalf of the Cochrane Bias Methods Group. Section 10.4 Detecting reporting biases. In: Higgins, JPT.; Green, S., editors. Cochrane Handbook for Systematic Reviews of Interventions version 5.01 (updated Sept 2008). The Cochrane Collaboration; 2008.

Taylor 1996 . Taylor E, Chadwick O, Heptinstall E, Danckaerts M. Hyperactivity and Conduct Problems as Risk Factors for Adolescent Development. Journal of the American Academy of Child \& Adolescent Psychiatry. 1996; 35(9):1213-26. [PubMed: 8824065]

Taylor 2004 . Taylor E, Döpfner M, Sergeant J, Asherson P, Banaschewski T, Buitelaar J, et al. European clinical guidelines for hyperkinetic disorder - first upgrade. European Child \& Adolescent Psychiatry. 2004; 13(Supplement 1):7-30.

Webster-Stratton 1998 . Webster-Stratton, C.; Hancock, L. Parent training for young children with conduct problems. Content, methods and therapeutic process. In: Schaefer, CE., editor. Handbook of parent training. Wiley; New York: 1998.

Weiss 1985 . Weiss G, Hechtman L, Milroy T, Perlman T. Psychiatric status of hyperactives as adults: A controlled prospective 15-year follow-up of 63 hyperactive children. Journal of the American Academy of Child \& Adolescent Psychiatry. 1985; 24(2):211-20.

Wells 2000 . Wells K, Pelham W, Kotkin R, Hoza B, Abikoff HB, Abramowitz A, et al. Psychosocial treatment strategies in the MTA study: rationale, methods, and critical issues in design and implementation. Journal of Abnormal Child Psychology. 2000; 28(6):483-505. [PubMed: 11104313]

Whitehead 1994 . Whitehead A, Jones NM. A meta-analysis of clinical trials involving different classifications of response into ordered categories. Statistics in Medicine. 1994; 13(23-24):250315. [MEDLINE: 95215666]. [PubMed: 7701150]

WHO 1977 . World Health Organisation. International Statistical Classification of Diseases and Related Health Problems, 9th Revision (ICD-9). World Health Organisation; Geneva: 1977.

WHO 1992 . World Health Organisation. International Statistical Classification of Diseases and Related Health Problems, 10th Revision (ICD-10). World Health Organisation; Geneva: 1992.

Yusuf 1991 . Yusuf S, Wittes J, Probstfield J, Tyroler HA. Analysis and interpretation of treatment effects in subgroups of patients in randomized clinical trials. JAMA. 1991; 266(1):93-8. [PubMed: 2046134]

* Indicates the major publication for the study 